\title{
INTEGRO-DIFFERENTIAL PROBLEM ABOUT PARAMETRIC AMPLIFICATION AND ITS ASYMPTOTICAL INTEGRATION
}

\author{
Abdukhafiz A. Bobodzhanov ${ }^{1}$, Burkhan T. Kalimbetov ${ }^{2}$, \\ Valery F. Safonov ${ }^{3}$ \\ 1 The National Research University, MPEI \\ Krasnokazarmennaya st. 14, Moscow, 111250, RUSSIA \\ ${ }^{2}$ Kh.A. Yasawi International Kazakh-Turkish University \\ Sattarkhanov ave. 29 \\ Turkestan, 161200, KAZAKHSTAN \\ 3 The National Research University, MPEI \\ Krasnokazarmennaya st. 14, Moscow, 111250, RUSSIA
}

\begin{abstract}
Asymptotic integration of differential systems of equations with fast oscillating coefficients has been carried out by the Feschenko-Shkil-Nikolenko splitting method and the Lomov regularization method. Equations of this type are often encountered in study of various questions related to dynamic stability, to properties of media with a periodic structure and other applied problems. In the monograph by Yu.L. Daletski and M.G. Krein an asymptotic analysis is given for one of these problems - the problem on parametric amplification. In the present paper, we generalize this problem to integro-differential equations, the differential part of which coincides with the parametric amplification problem. The main purpose of the research is to identify the influence of the integral term in the asymptotic behavior of the solution. It is considered the general case, i.e. the case of both the lack of resonance (when the integer linear combination of frequencies of the fast oscillating cosine does not coincide with the spectrum frequency of the limit operator), and its presence (when such coincidence takes place). The developed algorithm is obviously generalized to systems of equations with an arbitrary matrix of the differential part,
\end{abstract}

Received: November 22, 2019

(C) 2020 Academic Publications

$\S$ Correspondence author 
the pure imaginary spectrum, and with an arbitrary number of fast oscillating coefficients (such as the cosine considered in the paper).

AMS Subject Classification: 34K26, 45J05

Key Words: singularly perturbed; integro-differential equations; fast oscillating coefficients; regularization; asymptotic convergence

\section{Introduction}

The first of the problems with an irregular dependence in perturbation theory that arose in connection with the problems of celestial mechanics and electrical engineering were nonlinear equations, which are often called oscillating equations at present. Problems of this kind arise everywhere where certain transient processes take place. Studies of oscillating and singularly perturbed oscillating systems described by ordinary differential equations to the splitting methods were carried out in Yu. Daletskiy, M. Krein, S. Feschenko, N. Shkill, L. Nikolenko (see, [1],[2],[3]) and regularization methods in S. lomov, I. lomov, A. Ryzhih (see, [5],[6],[7],[8]). Singularly perturbed integro-differential systems with fast oscillating coefficients from the perspective of the regularization method were studied in B. Kalimbetov, V. Safonov [4], in which an algorithm for constructing asymptotic solutions was developed. The case of the absence of resonance is considered, i.e. when the integer linear combination of frequencies of the fast oscillating cosine does not coincide with the spectrum frequency of the limit operator. In this paper, we consider the resonance case.

Consider the following integro-differential system:

$$
\begin{gathered}
\varepsilon \frac{d z}{d t}-A(t) z-\varepsilon g(t) \cos \frac{\gamma(t)}{\varepsilon} B(t) z-\int_{t_{0}}^{t} K(t, s) z(s, \varepsilon) d s \\
=h(t), z\left(t_{0}, \varepsilon\right)=z^{0}, t \in\left[t_{0}, T\right]
\end{gathered}
$$

where $z=\left\{z_{1}(t, \varepsilon), z_{2}(t, \varepsilon)\right\}, h(t)=\left\{h_{1}(t), h_{2}(t)\right\}, \gamma^{\prime}(t)>0, \omega(t)>0$ $\left(\forall t \in\left[t_{0}, T\right]\right), g(t)$ - is a scalar function, $A(t)$ and $B(t)$ are $(2 \times 2)$ - matrices, moreover, $A(t)=\left(\begin{array}{rr}0 & 1 \\ -\omega^{2}(t) & 0\end{array}\right), z^{0}=\left\{z_{1}^{0}, z_{2}^{0}\right\}, \varepsilon>0$ a small parameter. Such a system was considered in [1-3] in the case of $\gamma(t)=2 \beta(t), B(t)=$ $\left(\begin{array}{ll}0 & 0 \\ 1 & 0\end{array}\right)$ and the absence of an integral term. The limit operator $A(t)$ has the spectrum $\lambda_{1}(t)=-i \omega(t), \lambda_{2}(t)=+i \omega(t)$, the frequency of the fast oscillating cosine is $\gamma^{\prime}(t)$. Furthermore, the functions $\lambda_{3}(t)=-i \gamma^{\prime}(t), \lambda_{4}(t)=+i \gamma^{\prime}(t)$ are said to be the spectrum of the fast oscillating coefficient. 
We consider the problem (1) under the following conditions:

$$
\begin{aligned}
& \text { 1) } \omega(t), \gamma(t), g(t) \in C^{\infty}\left(\left[t_{0}, T\right], C^{1}\right), h(t) \in C^{\infty}\left(\left[t_{0}, T\right], C^{2}\right), \\
& B(t) \in C^{\infty}\left(\left[t_{0}, T\right], C^{2 \times 2}\right), K(t, s) \in C^{\infty}\left(\left[t_{0}, T\right], C^{2 \times 2}\right),
\end{aligned}
$$

2) equalities

$$
\begin{aligned}
& n_{3} \lambda_{3}(t)+n_{4} \lambda_{4}(t)=\lambda_{j}(t), \\
& \lambda_{k}(t)+n_{3} \lambda_{3}(t)+n_{4} \lambda_{4}(t)=\lambda_{j}(t)
\end{aligned}
$$

either do not hold at any $t \in\left[t_{0}, T\right]$, or are fulfilled identically at all $t \in\left[t_{0}, T\right]$ for all multi-indices $n=\left(n_{3}, n_{4}\right)$ with $|n| \equiv n_{3}+n_{4} \geq 1$ (here $n_{3}$ and $n_{4}$ are integer nonnegative numbers) and $k, j=1,2$.

Under the given conditions, we try to develop the algorithm for constructing a regularized asymptotic solution [5] of the problem (1). Conditions 2) mean that the problem (1) will be studied both in the non-resonant case and in the presence of resonance.

\section{Regularization of the problem (1)}

By $\sigma_{j}=\sigma_{j}(\varepsilon)$, we denote the expressions $\sigma_{1}=e^{-\frac{i}{\varepsilon} \gamma\left(t_{0}\right)}, \sigma_{2}=e^{+\frac{i}{\varepsilon} \gamma\left(t_{0}\right)}$, which do not depend on $t$, and rewrite the problem (1) as

$$
\begin{gathered}
\varepsilon \frac{d z}{d t}-A(t) z-\varepsilon \frac{g(t)}{2}\left(e^{\frac{1}{\varepsilon} \int_{t_{0}}^{t} \lambda_{3}(\theta) d \theta} \sigma_{1}+e^{\frac{1}{\varepsilon} \int_{t_{0}}^{t} \lambda_{4}(\theta) d \theta} \sigma_{2}\right) B(t) z \\
-\int_{t_{0}}^{t} K(t, s) z(s, \varepsilon) d s=h(t), z\left(t_{0}, \varepsilon\right)=z^{0}, t \in\left[t_{0}, T\right] .
\end{gathered}
$$

We introduce regularizing variables

$$
\tau_{j}=\frac{1}{\varepsilon} \int_{t_{0}}^{t} \lambda_{j}(\theta) d \theta \equiv \frac{\psi_{j}(t)}{\varepsilon}, j=\overline{1,4},
$$

and instead of the problem (2) we consider the problem

$$
\begin{aligned}
& \varepsilon \frac{\partial \tilde{z}}{\partial t}+\sum_{j=1}^{4} \lambda_{j}(t) \frac{\partial \tilde{z}}{\partial \tau_{j}}-A(t) \tilde{z}-\varepsilon \frac{g(t)}{2}\left(e^{\tau_{3}} \sigma_{1}+e^{\tau_{4}} \sigma_{2}\right) B(t) \tilde{z} \\
& -\int_{t_{0}}^{t} K(t, s) \tilde{z}\left(s, \frac{\psi(s)}{\varepsilon}, \varepsilon\right) d s=h(t),\left.\tilde{z}(t, \tau, \varepsilon)\right|_{t=t_{0}, \tau=0}=z^{0},
\end{aligned}
$$


for the function $\tilde{z}=\tilde{z}(t, \tau, \varepsilon)$, where $\tau=\left(\tau_{1}, \tau_{2}, \tau_{3}, \tau_{4}\right), \quad \psi=\left(\psi_{1}, \psi_{2}, \psi_{3}, \psi_{4}\right)$ (see, (3)). It is clear that if $\tilde{z}=\tilde{z}(t, \tau, \varepsilon)$ is a solution of the problem (4), then the vector function $z=\tilde{z}\left(t, \frac{\psi(t)}{\varepsilon}, \varepsilon\right)$ is the exact solution of the problem (2), therefore the problem (4) is extended in relation to the problem (2). However, it cannot be considered completely regularized, since the integral term $J \tilde{z}=\int_{t_{0}}^{t} K(t, s) \tilde{z}\left(s, \frac{\psi(s)}{\varepsilon}, \varepsilon\right) d s$ is not regularized in it. To regularize the integral operator we introduce the class, asymptotically invariant with respect to the operator $J \tilde{z}$ (see [5], p. 62).

To do it we introduce a space $U$ of vector functions $z(t, \tau)$ represented as sums

$$
\begin{gathered}
z(t, \tau, \sigma)=z_{0}(t, \sigma)+\sum_{i=1}^{4} z_{i}(t, \sigma) e^{\tau_{i}}+\sum_{2 \leq|m| \leq N_{z}}^{*} z^{m}(t, \sigma) e^{(m, \tau)} \\
+\sum_{k=1}^{2} \sum_{1 \leq|m| \leq N_{z}}^{*} z^{e_{k}+m}(t, \sigma) e^{\left(e_{k}+m, \tau\right)}, \\
z_{i}(t, \sigma), z^{m}(t, \sigma), z^{e_{k}+m}(t, \sigma) \in C^{\infty}\left(\left[t_{0}, T\right], C^{2}\right), k=1,2, \\
m=\left(0,0, m_{3}, m_{4}\right), 1 \leq|m| \equiv m_{3}+m_{4} \leq N_{z}, i=\overline{1,4},
\end{gathered}
$$

where $(m, \lambda(t)) \equiv m_{3} \lambda_{3}(t)+m_{4} \lambda_{4}(t),\left(e_{j}+m, \lambda(t)\right) \equiv \lambda_{j}(t)+m_{3} \lambda_{3}(t)+$ $m_{4} \lambda_{4}(t)$; the asterisk $*$ above the sum sign indicates that the summation in it occurs only by non-resonant multi-indices, i.e. by $m=\left(0,0, m_{3}, m_{4}\right)$ such that

$$
\begin{aligned}
& m_{3} \lambda_{3}(t)+m_{4} \lambda_{4}(t) \neq 0, m_{3} \lambda_{3}(t)+m_{4} \lambda_{4}(t) \neq \lambda_{j}(t), \quad \lambda_{k}(t) \\
& +m_{3} \lambda_{3}(t)+m_{4} \lambda_{4}(t) \neq \lambda_{j}(t), \quad|m| \geq 1, j, k=1,2, \forall t \in\left[t_{0}, T\right],
\end{aligned}
$$

(here and further we will denote $\left.e_{1}=(1,0,0,0), e_{2}=(0,1,0,0), \sigma=\left(\sigma_{1}, \sigma_{2}\right)\right)$.

We note that here power $N_{z}$ of the polynomial $z(t, \tau, \sigma)$ with respect to the exponent $e^{\tau_{j}}$ depend on the element $z$. Moreover, elements of the space $U$ depend on bounded by $\varepsilon>0$ constants $\sigma_{1}=\sigma_{1}(\varepsilon)$ and $\sigma_{2}=\sigma_{2}(\varepsilon)$ which do not influence to development of the algorithm described below, therefore henceforth, in the record of the element (5) of the space $U$, for brevity, we omit the dependence on $\sigma=\left(\sigma_{1}, \sigma_{2}\right)$. We show that the class $M_{\varepsilon}=\left.U\right|_{\tau=\psi(t) / \varepsilon}$ is asymptotically invariant with respect to the operator $J$. The image of the operator $J$ on the element (5) of the space $U$ has the form

$$
J z(t, \varepsilon)=\int_{t_{0}}^{t} K(t, s) z_{0}(s) d s+\sum_{i=1}^{4} \int_{t_{0}}^{t} K(t, s) z_{i}(s) e^{\frac{1}{\varepsilon} \int_{t_{0}}^{s} \lambda_{j}(s) d \theta} d s
$$




$$
\begin{gathered}
+\sum_{2 \leq|m| \leq N_{z}}^{*} \int_{t_{0}}^{t} K(t, s) z^{m}(s) e^{\frac{1}{\varepsilon} \int_{t_{0}}^{s}(m, \lambda(\theta)) d \theta} d s \\
+\sum_{j=1}^{2} \sum_{1 \leq|m| \leq N_{z}}^{*} \int_{t_{0}}^{t} K(t, s) z^{e_{j}+m}(s) e^{\frac{1}{\varepsilon} \int_{t_{0}}^{s}\left(e_{j}+m, \lambda(\theta)\right) d \theta} d s .
\end{gathered}
$$

Integrating by parts, we will have

$$
\begin{aligned}
& J_{i}(t, \varepsilon)=\varepsilon \int_{t_{0}}^{t} \frac{K(t, s) z_{i}(s)}{\lambda_{i}(s)} d e^{\frac{1}{\varepsilon} \int_{t_{0}}^{s} \lambda_{i}(s) d \theta} \\
& =\varepsilon\left[\frac{K(t, t) z_{i}(t)}{\lambda_{i}(t)} e^{\frac{1}{\varepsilon} \int_{t_{0}}^{t} \lambda_{i}(s) d \theta}-\frac{K\left(t, t_{0}\right) z_{i}\left(t_{0}\right)}{\lambda_{i}\left(t_{0}\right)}\right] \\
& -\varepsilon \int_{t_{0}}^{t}\left(\frac{\partial}{\partial s} \frac{K(t, s) z_{i}(s)}{\lambda_{i}(s)}\right) e^{\frac{1}{\varepsilon} \int_{t_{0}}^{s} \lambda_{i}(s) d \theta} d s .
\end{aligned}
$$

Continuing this process further, we obtain the following decom-position

$$
\begin{gathered}
J_{i}(t, \varepsilon)=\sum_{\nu=0}^{\infty}(-1)^{\nu} \varepsilon^{\nu+1}\left[\left(I_{i}^{\nu}\left(K(t, s) z_{i}(s)\right)\right)_{s=t} e^{\left.\frac{1}{\varepsilon} \int_{t_{0}}^{t} \lambda_{i}(\theta)\right) d \theta}\right. \\
\left.-\left(I_{i}^{\nu}\left(K(t, s) z_{i}(s)\right)\right)_{s=t_{0}}\right],
\end{gathered}
$$

where $I_{i}^{0}=\frac{1}{\lambda_{i}(s)} \cdot, I_{i}^{\nu}=\frac{1}{\lambda_{i}(s)} I_{i}^{\nu-1}(\nu \geq 1, \quad i=\overline{1,4})$.

Applying the integration operation by parts to the integrals

$$
\begin{gathered}
J_{m}(t, \varepsilon)=\int_{t_{0}}^{t} K(t, s)(s) e^{\frac{1}{\varepsilon} \int_{t_{0}}^{s}(m, \lambda(\theta)) d \theta} d s \\
=\varepsilon \int_{t_{0}}^{t} \frac{K(t, s) z^{m}(s)}{(m, \lambda(s))} d e^{\frac{1}{\varepsilon} \int_{t_{0}}^{s}(m, \lambda(\theta)) d \theta} \\
=\varepsilon\left[\frac{K(t, t) z^{m}(t)}{(m, \lambda(t))} e^{\frac{1}{\varepsilon} \int_{t_{0}}^{t}(m, \lambda(\theta)) d \theta}-\frac{K\left(t, t_{0}\right) z^{m}\left(t_{0}\right)}{\left(m, \lambda\left(t_{0}\right)\right)}\right]
\end{gathered}
$$




$$
\begin{gathered}
-\varepsilon \int_{t_{0}}^{t} \frac{K(t, s) z^{m}(s)}{(m, \lambda(s))} e^{\frac{1}{\varepsilon} \int_{t_{0}}^{s}(m, \lambda(\theta)) d \theta} d s \\
=\sum_{\nu=0}^{\infty}(-1)^{\nu} \varepsilon^{\nu+1}\left[\left(I_{m}^{\nu}\left(K(t, s) z^{m}(s)\right)\right)_{s=t} e^{\frac{1}{\varepsilon} \int_{t_{0}}^{t}(m, \lambda(\theta)) d \theta}\right. \\
\left.-\left(I_{m}^{\nu}\left(K(t, s) z^{m}(s)\right)\right)_{s=t_{0}}\right]
\end{gathered}
$$

where $I_{m}^{0}=\frac{1}{(m, \lambda(s))} \cdot, I_{m}^{\nu}=\frac{1}{(m, \lambda(s))} \frac{\partial}{\partial s} I_{m}^{\nu-1}(\nu \geq 1,|m| \geq 2)$, (here it is taken into account that for elements of the space $U$ the inequalities $(m, \lambda(t)) \equiv$ $m_{3} \lambda_{3}(t)+m_{4} \lambda_{4}(t) \neq 0$ hold $)$;

$$
\begin{gathered}
J_{e_{j}+m}(t, \varepsilon)=\varepsilon \int_{t_{0}}^{t} \frac{K(t, s) z^{e_{k}+m}(s)}{\left(e_{k}+m, \lambda(s)\right)} d e^{\frac{1}{\varepsilon} \int_{t_{0}}^{s}\left(e_{k}+m, \lambda(\theta)\right) d \theta} \\
=\varepsilon\left[\frac{K(t, t) z^{e_{k}+m}(t)}{\left(e_{k}+m, \lambda(t)\right)} e^{\frac{1}{\varepsilon} \int_{t_{0}}^{t}\left(e_{k}+m, \lambda(\theta)\right) d \theta}-\frac{K\left(t, t_{0}\right) z^{e_{k}+m}\left(t_{0}\right)}{\left(e_{k}+m, \lambda\left(t_{0}\right)\right)}\right] \\
-\varepsilon \int_{t_{0}}^{t} \frac{\partial}{\partial s} \frac{K(t, s) z^{e_{k}+m}(s)}{\left(e_{k}+m, \lambda(s)\right)} e^{\frac{1}{\varepsilon} \int_{t_{0}}^{s}\left(e_{k}+m, \lambda(\theta)\right) d \theta} d s \\
=\sum_{\nu=0}^{\infty}(-1)^{\nu} \varepsilon^{\nu+1}\left[\begin{array}{l}
\left(I_{k, m}^{\nu}\left(K(t, s) z^{e_{k}+m}(s)\right)\right)_{s=t} e^{\frac{1}{\varepsilon} \int_{t_{0}}^{t}\left(e_{k}+m, \lambda(\theta)\right) d \theta} \\
\left.-\left(I_{k, m}^{\nu}\left(K(t, s) z^{e_{k}+m}(s)\right)\right)_{s=t_{0}}\right]
\end{array}\right.
\end{gathered}
$$

where $I_{k, m}^{0}=\frac{1}{\left(e_{k}+m, \lambda(s)\right)} \cdot, \quad I_{k, m}^{\nu}=\frac{1}{\left(e_{k}+m, \lambda(s)\right)} \frac{\partial}{\partial s} I_{k, m}^{\nu-1} \quad(\nu \geq 1$, $|m| \geq 1, k=1,2)$, here it is taken into account that for elements of the space $U$ the inequalities $(m, \lambda(t)) \equiv\left(e_{k}+m, \lambda(t)\right) \equiv \lambda_{k}(t)+m_{3} \lambda_{3}(t)+m_{4} \lambda_{4}(t) \neq$ $0, k=1,2$, hold. Indeed, if for a some $t \in\left[t_{0}, T\right]$ and $|m| \geq 1$ it is true that $\lambda_{k}(t)+m_{3} \lambda_{3}(t)+m_{4} \lambda_{4}(t)=0, k \in\{1,2\}$, then $\lambda_{k}(t)+m_{3} \lambda_{3}(t)+$ $m_{4} \lambda_{4}(t)=0 \Leftrightarrow m_{3} \lambda_{3}(t)+m_{4} \lambda_{4}(t)=-\lambda_{k}(t)=\lambda_{j}(t), j \neq k$, i.e. multi-index $m=\left(0,0, m_{3}, m_{4}\right)$ would be resonant, which contradicts the definition of the 
class $U$. Hence, the image of the operator $J$ on the element (5) of space $U$ is represented as a series

$$
\begin{aligned}
& J z(t, \tau)=\int_{t_{0}}^{t} K(t, s) z_{0}(s) d s \\
& +\sum_{i=1}^{4} \sum_{\nu=0}^{\infty}(-1)^{\nu} \varepsilon^{\nu+1}\left[\left(I_{i}^{\nu}\left(K(t, s) z_{i}(s)\right)\right)_{s=t} e^{\left.\frac{1}{\varepsilon} \int_{t_{0}}^{t} \lambda_{i}(\theta)\right) d \theta}\right. \\
& \text { - } \left.\left(I_{i}^{\nu}\left(K(t, s) z_{i}(s)\right)\right)_{s=t_{0}}\right] \\
& +\sum_{2 \leq|m| \leq N_{z}}^{*} \sum_{\nu=0}^{\infty}(-1)^{\nu} \varepsilon^{\nu+1}\left[\left(I_{m}^{\nu}\left(K(t, s) z^{m}(s)\right)\right)_{s=t} e^{\frac{1}{\varepsilon} \int_{t_{0}}^{t}(m, \lambda(\theta)) d \theta}\right. \\
& \left.-\left(I_{m}^{\nu}\left(K(t, s) z^{m}(s)\right)\right)_{s=t_{0}}\right] \\
& +\sum_{k=1}^{2} \sum_{1 \leq|m| \leq N_{z}}^{*}(-1)^{\nu} \varepsilon^{\nu+1}\left[\left(I_{k, m}^{\nu}\left(K(t, s) z^{e_{k}+m}(s)\right)\right)_{s=t} \times\right. \\
& \left.\times e^{\frac{1}{\varepsilon} \int_{t_{0}}^{t}\left(e_{k}+m, \lambda(\theta)\right) d \theta}-\left(I_{k, m}^{\nu}\left(K(t, s) z^{e_{k}+m}(s)\right)\right)_{s=t_{0}}\right], \nu \geq 1 .
\end{aligned}
$$

It is easy to show (see, e.g., [9], p. 291-294), that this series converges asymptotically as $\varepsilon \rightarrow+0$ (uniformly by $t \in\left[t_{0}, T\right]$ ). It means that the class $M_{\varepsilon}$ is asymptotically invariant (as $\varepsilon \rightarrow+0$ ) with respect to the operator $J$.

We introduce the operators $R_{\nu}: U \rightarrow U$, acting on each element $z(t, \tau) \in U$ of the form (5) by the law:

$$
\begin{gathered}
R_{0} z(t, \tau)=\int_{t_{0}}^{t} K(t, s) z_{0}(s) d s \\
R_{1} z(t, \tau)=\sum_{i=1}^{4}\left[\left(I_{i}^{0}\left(K(t, s) z_{i}(s)\right)\right)_{s=t} e^{\tau_{i}}\right. \\
\left.-\left(I_{i}^{0}\left(K(t, s) z_{i}(s)\right)\right)_{s=t_{0}}\right] \\
+\sum_{2 \leq|m| \leq N_{z}}^{*}\left[\left(I_{m}^{0}\left(K(t, s) z^{m}(s)\right)\right)_{s=t} e^{(m, \tau)}\right. \\
\left.-\left(I_{m}^{0}\left(K(t, s) z^{m}(s)\right)\right)_{s=t_{0}}\right]
\end{gathered}
$$




$$
\begin{gathered}
+\sum_{k=1}^{2} \sum_{1 \leq|m| \leq N_{z}}^{*}\left[\left(I_{k, m}^{0}\left(K(t, s) z^{e_{k}+m}(s)\right)\right)_{s=t} e^{\left(e_{k}+m, \tau\right)}\right. \\
\left.-\left(I_{k, m}^{0}\left(K(t, s) z^{e_{k}+m}(s)\right)\right)_{s=t_{0}}\right] \\
R_{\nu+1} z(t, \tau)=\sum_{i=1}^{4}(-1)^{\nu} \varepsilon^{\nu+1}\left[\left(I_{i}^{\nu}\left(K(t, s) z_{i}(s)\right)\right)_{s=t} e^{\tau_{i}}\right. \\
\left.-\left(I_{i}^{\nu}\left(K(t, s) z_{i}(s)\right)\right)_{s=t_{0}}\right] \\
+\sum_{\nu=0}^{*}(-1)^{\nu} \varepsilon^{\nu+1}\left[\left(I_{m}^{\nu}\left(K(t, s) z^{m}(s)\right)\right)_{s=t} e^{(m, \tau)}\right. \\
\left.-\left(I_{m}^{\nu}\left(K(t, s) z^{m}(s)\right)\right)_{s=t_{0}}\right] \\
+\sum_{k=1}^{2} \quad(-1)^{\nu}\left[\left(I_{k, m}^{\nu}\left(K(t, s) z^{e_{k}+m}(s)\right)\right)_{s=t} e^{\left(e_{k}+m, \tau\right)}\right. \\
\left.-\left(I_{k, m}^{\nu}\left(K(t, s) z^{e_{k}+m}(s)\right)\right)_{s=t_{0}}\right], \nu \geq 1 .
\end{gathered}
$$

Let now $\tilde{z}(t, \tau, \varepsilon)$ be an arbitrary continuous by

$$
(t, \tau) \in\left[t_{0}, T\right] \times\left\{\tau: \operatorname{Re} \tau_{j} \leq 0, j=\overline{1,4}\right\}
$$

function, having the asymptotic decomposition:

$$
\tilde{z}(t, \tau, \varepsilon)=\sum_{k=0}^{\infty} \varepsilon^{k} z_{k}(t, \tau), z_{k}(t, \tau) \in U
$$

which converges as $\varepsilon \rightarrow+0$ (uniformly by $(t, \tau) \in\left[t_{0}, T\right] \times$ $\left\{\tau: \operatorname{Re} \tau_{j} \leq 0, j=\overline{1,4}\right\}$. Then the image of the operator $J \tilde{z}(t, \tau, \varepsilon)$ of this function expands into asymptotic series

$$
J \tilde{z}(t, \tau, \varepsilon)=\sum_{k=0}^{\infty} \varepsilon^{k} J z_{k}(t, \tau)=\left.\sum_{r=0}^{\infty} \varepsilon^{r} \sum_{s=0}^{r} R_{r-s} z_{s}(t, \tau)\right|_{\tau=\psi(t) / \varepsilon} .
$$

This equality is the basis to introduce an expansion of the operator $J$ on the series of the form (7):

$$
\tilde{J} \tilde{z}(t, \tau, \varepsilon) \equiv \tilde{J}\left(\sum_{k=0}^{\infty} \varepsilon^{k} z_{k}(t, \tau)\right) \triangleq \sum_{r=0}^{\infty} \varepsilon^{r} \sum_{s=0}^{r} R_{r-s} z_{s}(t, \tau) .
$$

Although the operator $\tilde{J}$ is formally defined, its utility is obvious, since in practice the $N$ - th approximation of the asymptotic solution (2) is usually built, in which only the $N$ - th partial sums of the series (7) will be involved, 
which have a non-formal, but a true meaning. Now we can write a problem that is completely regularized with respect to the original problem (2):

$$
\begin{gathered}
\varepsilon \frac{\partial \tilde{z}}{\partial t}+\sum_{j=1}^{4} \lambda_{j}(t) \frac{\partial \tilde{z}}{\partial \tau_{j}}-A(t) \tilde{z}-\varepsilon \frac{g(t)}{2}\left(e^{\tau_{3}} \sigma_{1}+e^{\tau_{4}} \sigma_{2}\right) B \tilde{z} \\
-\tilde{J} \tilde{z}=h(t),\left.\quad \tilde{z}(t, \tau, \varepsilon)\right|_{t=t_{0}, \tau=0}=z^{0}, \quad t \in\left[t_{0}, T\right] .
\end{gathered}
$$

\section{Iteration problems and their solvability in the space $U$. Solution of the first iteration problem}

Substituting the series (7) into (9) and equating the coefficients at the same degrees of $\varepsilon$, we obtain the following iteration problems:

$$
\begin{gathered}
L z_{0}(t, \tau) \equiv \sum_{j=1}^{4} \lambda_{j}(t) \frac{\partial z_{0}}{\partial \tau_{j}}-A(t) z_{0}-R_{0} z_{0}=h(t), \\
z_{0}\left(t_{0}, 0\right)=z^{0} \\
L z_{1}(t, \tau)=-\frac{\partial z_{0}}{\partial t}+\frac{g(t)}{2}\left(e^{\tau_{3}} \sigma_{1}+e^{\tau_{4}} \sigma_{2}\right) B(t) z_{0} \\
+R_{1} z_{0}, \quad z_{1}\left(t_{0}, 0\right)=0 \\
L z_{2}(t, \tau)=-\frac{\partial z_{1}}{\partial t}+\frac{g(t)}{2}\left(e^{\tau_{3}} \sigma_{1}+e^{\tau_{4}} \sigma_{2}\right) B(t) z_{1} \\
+R_{1} z_{1}+R_{2} z_{0}, \quad z_{0}\left(t_{0}, 0\right)=0 \\
L \begin{array}{c}
L z_{k}(t, \tau)=-\frac{\partial z_{k-1}}{\partial t}+\frac{g(t)}{2}\left(e^{\tau_{3}} \sigma_{1}+e^{\tau_{4}} \sigma_{2}\right) B(t) z_{k-1} \\
+R_{k} z_{0}+\ldots+R_{1} z_{k-1}, \quad z_{k}\left(t_{0}, 0\right)=0, k \geq 1 .
\end{array}
\end{gathered}
$$

Each of the iteration problems $\left(10_{k}\right)$ can be written in the form:

$$
\begin{gathered}
L z(t, \tau) \equiv \sum_{j=1}^{4} \lambda_{j}(t) \frac{\partial z}{\partial \tau_{j}}-A(t) z-R_{0} z=H(t, \tau), \\
z\left(t_{0}, 0\right)=z^{*}
\end{gathered}
$$

where $H(t, \tau)=H_{0}(t)+\sum_{i=1}^{4} H_{i}(t) e^{\tau_{i}}+\sum_{2 \leq|m| \leq N_{z}}^{*} H^{m}(t) e^{(m, \tau)}$

$+\sum_{k=1}^{2} \sum_{1 \leq|m| \leq N_{H}}^{*} H^{e_{k}+m}(t) e^{\left(e_{k}+m, \tau\right)}$ is known vector function of the space $U, z^{*}$ 
is known constant vector of the complex space $C^{2}$, and the operator $R_{0}$ has the form $\left(\right.$ see $\left.\left(6_{0}\right)\right)$

$$
\begin{aligned}
& R_{0} z \equiv R_{0}\left(z_{0}(t)+\sum_{i=1}^{4} z_{i}(t) e^{\tau_{i}}+\sum_{2 \leq|m| \leq N_{z}}^{*} z^{m}(t) e^{(m, \tau)}\right. \\
& \left.+\sum_{k=1}^{2} \sum_{0 \leq|m| \leq N_{z}}^{*} z^{e_{k}+m}(t) e^{\left(e_{k}+m, \tau\right)}\right) \triangleq \int_{t_{0}}^{t} K(t, s) z_{0}(s) d s .
\end{aligned}
$$

Furthermore, we need $\lambda_{j}(t)$ eigenvectors of the matrix $A(t)$ :

$$
\varphi_{1}(t)=\left(\begin{array}{c}
1 \\
-i \omega(t)
\end{array}\right), \varphi_{2}(t)=\left(\begin{array}{c}
1 \\
+i \omega(t)
\end{array}\right),
$$

and $\bar{\lambda}_{j}(t)$ eigenvectors of the matrix $A^{*}(t)$ :

$$
\chi_{1}(t)=\left(\begin{array}{c}
1 \\
-\frac{i}{\omega(t)}
\end{array}\right), \chi_{2}(t)=\left(\begin{array}{c}
1 \\
+\frac{i}{\omega(t)}
\end{array}\right) .
$$

These vectors form a biorthogonal system, that is,

$$
\left(\varphi_{k}(t), \chi_{j}(t)\right)=\left\{\begin{array}{l}
2, k=j, \\
0, k \neq j,
\end{array} \quad(k, j=1,2) .\right.
$$

We introduce the scalar (at each $t \in\left[t_{0}, T\right]$ ) product in the space $U$ :

$$
\begin{aligned}
& <z, w>\equiv<z_{0}(t)+\sum_{i=1}^{4} z_{i}(t) e^{\tau_{i}}+\sum_{2 \leq|m| \leq N_{z}}^{*} z^{m}(t) e^{(m, \tau)} \\
& +\sum_{k=1}^{2} \sum_{1 \leq|m| \leq N_{z}}^{*} z^{e_{k}+m}(t) e^{\left(e_{l}+m, \tau\right)}, w_{0}(t)+\sum_{i=1}^{4} w_{i}(t) e^{\tau_{i}} \\
& +\sum_{k=1}^{2} \sum_{1 \leq|m| \leq N_{w}}^{*} w^{e_{k}+m}(t) e^{\left(e_{k}+m, \tau\right)}>\triangleq\left(z_{0}(t), w_{0}(t)\right) \\
& +\sum_{i=1}^{4}\left(z_{i}(t), w_{i}(t)\right)+\sum_{2 \leq|m| \leq \min \left(N_{z}, N_{w}\right)}^{*}\left(z^{m}(t), w^{m}(t)\right) \\
& +\sum_{k=1}^{2} \sum_{1 \leq|m| \leq \min \left(N_{z}, N_{w}\right)}^{*}\left(z^{e_{k}+m}(t), w^{e_{k}+m}(t)\right),
\end{aligned}
$$

where by $(*, *)$ we denote the usual scalar product in the complex space $C^{2}$. We prove the following proposition. 
Theorem 1. Let the conditions 1) and 2) hold and the right side $H(t, \tau)=$ $H_{0}(t)+\sum_{i=1}^{4} H_{i}(t) e^{\tau_{i}}+\sum_{2 \leq|m| \leq N_{z}}^{*} H^{m}(t) e^{(m, \tau)}$

$+\sum_{k=1}^{2} \sum_{1 \leq|m| \leq N_{H}}^{*} H^{e_{k}+m}(t) e^{\left(e_{k}+m, \tau\right)}$ of the system (11) belong to the space $U$. Then for solvability of the system (11) in $U$ it is necessary and sufficient the following identity

$$
<H(t, \tau), \chi_{j}(t) e^{\tau_{j}}>\equiv 0, j=1,2, \forall t \in\left[t_{0}, T\right] .
$$

Proof. We will define a solution of the system (11) in the form of the element (5) of the space $U$ :

$$
\begin{aligned}
& z(t, \tau)=z_{0}(t)+\sum_{i=1}^{4} z_{i}(t) e^{\tau_{i}}+\sum_{2 \leq|m| \leq N_{z}}^{*} z^{m}(t) e^{(m, \tau)} \\
& +\sum_{j=k}^{2} \sum_{1 \leq|m| \leq N_{H}}^{*} z^{e_{k}+m}(t) e^{\left(e_{k}+m, \tau\right)} \equiv z_{0}(t)+\sum_{i=1}^{4} z_{i}(t) e^{\tau_{i}} \\
& +\sum_{2 \leq|m| \leq N_{z}}^{*} z^{m}(t) e^{(m, \tau)}+\sum_{k=1}^{2} \sum_{2 \leq\left|m^{k}\right| \leq N_{H}}^{*} z^{m^{k}}(t) e^{\left(m^{k}, \tau\right)},
\end{aligned}
$$

where for convenience we introduce multi-indices $m^{k}$ :

$$
\begin{gathered}
m^{1}=e_{1}+m \equiv\left(1,0, m_{3}, m_{4}\right), \quad m^{2}=e_{2}+m \equiv\left(0,1, m_{3}, m_{4}\right), \\
\left|m^{k}\right|=1+m_{3}+m_{4} \geq 2,
\end{gathered}
$$

$m_{3}$ and $m_{4}$ are integer nonnegative numbers. Putting (13) into the system (11), we have

$$
\begin{gathered}
\sum_{i=1}^{4}\left[\lambda_{i}(t) I-A(t)\right] z_{i}(t) e^{\tau_{i}} \\
+\sum_{2 \leq|m| \leq N_{z}}^{*}[(m, \lambda(t)) I-A(t)] z^{m}(t) e^{(m, \tau)} \\
+\sum_{k=1}^{2} \sum_{2 \leq\left|m^{k}\right| \leq N_{H}}^{*}\left[\left(m^{k}, \lambda(t)\right) I-A(t)\right] z^{m^{k}}(t) e^{\left(m^{k}, \tau\right)}
\end{gathered}
$$




$$
\begin{gathered}
-A(t) z_{0}(t)-\int_{t_{0}}^{t} K(t, s) z_{0}(s) d s \\
=H_{0}(t)+\sum_{i=1}^{4} H_{i}(t) e^{\tau_{i}}+\sum_{2 \leq|m| \leq N_{z}}^{*} H^{m}(t) e^{(m, \tau)} \\
+\sum_{k=1}^{2} \sum_{2 \leq\left|m^{k}\right| \leq N_{H}}^{*} H^{m^{k}}(t) e^{\left(m^{k}, \tau\right) .}
\end{gathered}
$$

Equating here separately the free terms and coefficients at the same exponents, we obtain the following systems of equations:

$$
\begin{gathered}
-A(t) z_{0}(t)-\int_{t_{0}}^{t} K(t, s) z_{0}(s) d s=H_{0}(t), \\
{\left[\lambda_{i}(t) I-A(t)\right] z_{i}(t)=H_{i}(t), i=\overline{1,4},} \\
{[(m, \lambda(t)) I-A(t)] z^{m}(t)=H^{m}(t),} \\
(m, \lambda(t)) \neq \lambda_{j}(t), 2 \leq|m| \leq N_{H}, j=1,2, \\
{\left[\left(m^{k}, \lambda(t)\right) I-A(t)\right] z^{m^{k}}(t)=H^{m^{k}}(t),} \\
2 \leq\left|m^{k}\right| \leq N_{H}, m^{k}=e_{k}+m:\left(e_{k}+m, \lambda(t)\right) \\
\neq \lambda_{j}(t), 1 \leq|m| \leq N_{H}, k, j=1,2 .
\end{gathered}
$$

Since the matrix $A(t)$ is invertible, then the system (14) can be written in the following form

$$
z_{0}(t)=\int_{t_{0}}^{t}\left(-A^{-1}(t) K(t, s)\right) z_{0}(s) d s-A^{-1}(t) H_{0}(t) .
$$

Due to smoothness of the kernel $-A^{-1}(t) K(t, s)$ and non-homo-geneity of $-A^{-1}(t) H_{0}(t)$, this Voltera integral system has unique solution $z_{0}(t) \in C^{\infty}\left(\left[t_{0}, T\right], C^{2}\right)$. Systems $\left(14_{3}\right)-\left(14_{4}\right)$ also have unique solutions

$$
z_{i}(t)=\left[\lambda_{i}(t) I-A(t)\right]^{-1} H_{i}(t) \in C^{\infty}\left(\left[t_{0}, T\right], C^{2}\right), i=3,4,
$$

since $\lambda_{3}(t), \lambda_{4}(t)$ do not belong to spectrum of the matrix $A(t)$. Systems $\left(14_{1}\right)$ and $\left(14_{2}\right)$ are solvable in the space $C^{\infty}\left(\left[t_{0}, T\right], C^{2}\right)$ if and only if the identity 
$\left(H_{i}(t), \chi_{i}(t)\right) \equiv 0 \forall t \in\left[t_{0}, T\right], i=1,2$, holds. It is easy to see that these identities coincide with the identities (12).

Further, since $(m, \lambda(t)) \equiv m_{3} \lambda_{3}(t)+m_{4} \lambda_{4}(t) \neq \lambda_{j}(t), j=1,2,|m|=$ $m_{3}+m_{4} \geq 2, m^{k}=e_{k}+m:\left(e_{k}+m, \lambda(t)\right) \neq \lambda_{j}(t), 1 \leq|m| \leq N_{H}, k, j=$ 1,2 , (see the definition of the class $U$ ), then the systems $\left(14_{m}\right)$ and (15) have unique solutions

$$
\begin{gathered}
z^{m}(t)=[(m, \lambda(t)) I-A(t)]^{-1} H^{m}(t), 2 \leq|m| \leq N_{H}, \\
z^{m^{k}}(t)=\left[\left(m^{k}, \lambda(t)\right) I-A(t)\right]^{-1} H^{m^{k}}(t), \\
2 \leq\left|m^{k}\right| \leq N_{H}, k=1,2 .
\end{gathered}
$$

Thus, the condition (12) is necessary and sufficient for solvability of the system (12) in the space $U$. Theorem 1 is proved.

Remark. If the identity (12) holds, then with the conditions 1) and 2) the system (11) has the following solution in the space $U$ :

$$
\begin{aligned}
z(t, \tau) & =z_{0}(t)+\sum_{i=1}^{4} z_{i}(t) e^{\tau_{i}}+\sum_{2 \leq|m| \leq N_{z}}^{*} z^{m}(t) e^{(m, \tau)} \\
+ & \sum_{j=k}^{2} \sum_{1 \leq|m| \leq N_{H}}^{*} z^{e_{k}+m}(t) e^{\left(e_{k}+m, \tau\right)},
\end{aligned}
$$

where:

$$
\begin{gathered}
\sum_{i=1}^{4} z_{i}(t) e^{\tau_{i}} \equiv \sum_{k=1}^{2} \alpha_{k}(t) \varphi_{k}(t) e^{\tau_{k}}+\frac{\left(H_{1}(t), \chi_{2}(t)\right)}{\lambda_{1}(t)-\lambda_{2}(t)} \varphi_{2}(t) e^{\tau_{1}} \\
+\frac{\left(H_{2}(t), \chi_{1}(t)\right)}{\lambda_{2}(t)-\lambda_{1}(t)} \varphi_{1}(t) e^{\tau_{2}}+\sum_{i=3}^{4}\left[\lambda_{i}(t) I-A(t)\right]^{-1} H_{i}(t) e^{\tau_{i}}, \\
z^{m}(t) \equiv[(m, \lambda(t)) I-A(t)]^{-1} H^{m}(t), 2 \leq|m| \leq N_{H}, \\
z^{e_{k}+m}(t) \equiv\left[\left(e_{k}+m, \lambda(t)\right) I-A(t)\right]^{-1} H^{e_{k}+m}(t), \\
1 \leq|m| \leq N_{H}, k=1,2,
\end{gathered}
$$

more over, $\alpha_{k}(t) \in C^{\infty}\left(\left[t_{0}, T\right], C^{1}\right)$ are arbitrary functions, $k=1,2$, and $z_{0}(t)$ is a solution of the integral system $m \equiv\left(0,0, m_{3}, m_{4}\right),|m|=m_{3}+m_{4} \geq 1$. 


\section{Unique solvability of a general iteration problem in the space $U$. Residual term theorem}

Let us proceed to the description of conditions for unique solvability of the system (11) in the space $U$. Along with the problem (11), we consider the system

$$
L w(t, \tau)=-\frac{\partial z}{\partial t}+\frac{g(t)}{2}\left(e^{\tau_{3}} \sigma_{1}+e^{\tau_{4}} \sigma_{2}\right) B(t) z+Q(t, \tau),
$$

where $z=z(t, \tau)$ is a solution (16) of the system (11), $Q(t, \tau) \in U$ is known function in the space $U$. Right hand side of this system:

$$
\begin{gathered}
G(t, \tau) \equiv-\frac{\partial z}{\partial t}+\frac{g(t)}{2}\left(e^{\tau_{3}} \sigma_{1}+e^{\tau_{4}} \sigma_{2}\right) B(t) z+Q(t, \tau) \\
=-\frac{\partial}{\partial t}\left[z_{0}(t)+\sum_{i=1}^{4} z_{i}(t) e^{\tau_{i}}+\sum_{2 \leq|m| \leq N_{z}}^{*} z^{m}(t) e^{(m, \tau)}\right. \\
\left.+\sum_{k=1}^{2} \sum_{1 \leq|m| \leq N_{z}}^{*} z^{e_{k}+m}(t) e^{\left(e_{k}+m, \tau\right)}\right] \\
+\frac{g(t)}{2}\left(e^{\tau_{3}} \sigma_{1}+e^{\tau_{4}} \sigma_{2}\right) B(t)\left[z_{0}(t)+\sum_{i=1}^{4} z_{i}(t) e^{\tau_{i}}\right. \\
\left.+\sum_{2 \leq|m| \leq N_{z}}^{*} z^{m}(t) e^{(m, \tau)}+\sum_{k=1}^{2} \sum_{1 \leq|m| \leq N_{z}}^{*} z^{e_{k}+m}(t) e^{\left(e_{k}+m, \tau\right)}\right]+Q(t, \tau),
\end{gathered}
$$

can not belong to the space $U$, if $z=z(t, \tau) \in U$. Indeed, taking into account the form (16) of the function $z=z(t, \tau) \in U$, we will have

$$
\begin{gathered}
Z(t, \tau) \equiv G(t, \tau)+\frac{\partial z}{\partial t} \\
=\frac{g(t)}{2}\left(e^{\tau_{3}} \sigma_{1}+e^{\tau_{4}} \sigma_{2}\right) B(t)\left[z_{0}(t)+\sum_{i=1}^{4} z_{i}(t) e^{\tau_{i}}\right. \\
\left.+\sum_{2 \leq|m| \leq N_{z}}^{*} z^{m}(t) e^{(m, \tau)}+\sum_{k=1}^{2} \sum_{1 \leq|m| \leq N_{z}}^{*} z^{e_{k}+m}(t) e^{\left(e_{k}+m, \tau\right)}\right]
\end{gathered}
$$




$$
\begin{gathered}
+\frac{g(t)}{2} B(t) z_{0}(t)\left(e^{\tau_{3}} \sigma_{1}+e^{\tau_{4}} \sigma_{2}\right)+\sum_{i=3}^{4} \frac{g(t)}{2} B(t) z_{i}(t) \\
\times\left(e^{\tau_{i}+\tau_{3}} \sigma_{1}+e^{\tau_{i}+\tau_{4}} \sigma_{2}\right)+\sum_{k=1}^{2} \frac{g(t)}{2} B(t) z_{k}(t)\left(e^{\tau_{k}+\tau_{3}} \sigma_{1}+e^{\tau_{k}+\tau_{4}} \sigma_{2}\right) \\
+\frac{g(t)}{2} B(t) \sum_{2 \leq|m| \leq N_{z}}^{*}\left[z^{m}(t)\left(e^{\tau_{3}} \sigma_{1}+e^{\tau_{4}} \sigma_{2}\right) e^{(m, \tau)}\right. \\
\left.+\sum_{k=1}^{2} \sum_{1 \leq|m| \leq N_{z}}^{*} z^{e_{k}+m}(t)\left(e^{\tau_{3}} \sigma_{1}+e^{\tau_{4}} \sigma_{2}\right) e^{\left(e_{k}+m, \tau\right)}\right]+Q(t, \tau) .
\end{gathered}
$$

Here, for example, terms with exponents

$$
\begin{aligned}
& e^{\tau_{4}+\tau_{3}}=\left.e^{(m, \tau)}\right|_{m=(0,0,1,1)}, \quad e^{\tau_{3}+(m, \tau)}\left(m_{3}+1=m_{4}\right), \\
& e^{\tau_{4}+(m, \tau)}\left(m_{4}+1=m_{3}\right), \quad e^{\tau_{3}+\left(e_{1}+m, \tau\right)}\left(m_{3}+1=m_{4}\right), \\
& e^{\tau_{4}+\left(e_{2}+m, \tau\right)}\left(m_{4}+1=m_{3}\right)
\end{aligned}
$$

do not belong to the space $U$, since the multi-indices $m=(0,0, s, s), m=$ $(1,0, s, s), m=(0,1, s, s)(s \geq 1)$ are resonant. Then by the well-known theory (see [5], p. 234), it is necessary to make embedding $Z(t, \tau) \rightarrow \widehat{Z}(t, \tau)$ into the space $U$. To do it we introduce the following sets of resonant multi-indices:

$$
\begin{gathered}
\Gamma_{0}=\left\{m=\left(0,0, m_{3}, m_{4}\right):(m, \lambda(t)) \equiv 0,|m| \geq 1\right\}, \\
\Gamma_{k}=\left\{m=\left(0,0, m_{3}, m_{4}\right):(m, \lambda(t)) \equiv \lambda_{k}(t),|m| \geq 2\right\}, k=1,2, \\
\Gamma_{k, j}=\left\{m^{k}=e_{k}+\left(0,0, m_{3}, m_{4}\right):\left(e_{k}+m, \lambda(t)\right) \equiv \lambda_{j}(t)\right\}, \\
\left|m^{k}\right| \geq 1, k, j=1,2 .
\end{gathered}
$$

We denote

$$
\begin{aligned}
& \frac{g(t)}{2} B(t)\left[\sum_{2 \leq|m| \leq N_{z}}^{*} z^{m}(t)\left(e^{\tau_{3}} \sigma_{1}+e^{\tau_{4}} \sigma_{2}\right) e^{(m, \tau)}\right. \\
& \left.+\sum_{k=1}^{2} \sum_{1 \leq|m| \leq N_{z}}^{*} z^{e_{k}+m}(t)\left(e^{\tau_{3}} \sigma_{1}+e^{\tau_{4}} \sigma_{2}\right) e^{\left(e_{k}+m, \tau\right)}\right] \\
\equiv & \sum_{2 \leq|m| \leq N_{w}}^{*} w^{m}(t) e^{(m, \tau)}+\sum_{k=1}^{2} \sum_{1 \leq|m| \leq N_{w}}^{*} w^{e_{k}+m}(t) e^{\left(e_{k}+m, \tau\right)}
\end{aligned}
$$




$$
\begin{gathered}
+\sum_{2 \leq|m| \leq N_{w}: m \in \Gamma_{0}} w^{m}(t) e^{(m, \tau)}+\sum_{k=1}^{2} \sum_{2 \leq|m| \leq N_{w}: m \in \Gamma_{k}} w^{m}(t) e^{(m, \tau)} \\
+\sum_{k=1}^{2} \sum_{j=1}^{2} \sum_{1 \leq\left|m^{k}\right| \leq N_{w}: m^{k} \in \Gamma_{k, j}} w^{m^{k}}(t) e^{\left(m^{k}, \tau\right)}
\end{gathered}
$$

selecting in the left hand side the resonant and non-resonant parts with respect to the exponents $e^{\tau_{j}}, j=\overline{1,4}$. Then we can write $Z(t, \tau)$ in the form

$$
\begin{gathered}
Z(t, \tau)=\frac{g(t)}{2} B(t) z_{0}(t)\left(e^{\tau_{3}} \sigma_{1}+e^{\tau_{4}} \sigma_{2}\right) \\
+\sum_{i=3}^{4} \frac{g(t)}{2} B(t) z_{i}(t)\left(e^{\tau_{i}+\tau_{3}} \sigma_{1}+e^{\tau_{i}+\tau_{4}} \sigma_{2}\right) \\
+\sum_{k=1}^{2} \frac{g(t)}{2} B(t) z_{k}(t)\left(e^{\tau_{k}+\tau_{3}} \sigma_{1}+e^{\tau_{k}+\tau_{4}} \sigma_{2}\right) \\
+\sum_{2 \leq|m| \leq N_{w}}^{*} w^{m}(t) e^{(m, \tau)}+\sum_{k=1}^{2} \sum_{1 \leq|m| \leq N_{w}}^{*} w^{e_{k}+m}(t) e^{\left(e_{k}+m, \tau\right)} \\
+\sum_{2 \leq|m| \leq N_{w}: m \in \Gamma_{0}} w^{m}(t) e^{(m, \tau)}+\sum_{k=1}^{2} \sum_{2 \leq|m| \leq N_{w}: m \in \Gamma_{k}} w^{m}(t) e^{(m, \tau)} \\
+\sum_{k=1}^{2} \sum_{j=1}^{2} \sum_{1 \leq\left|m^{k}\right| \leq N_{w}: m \in \Gamma_{k, j}} w^{m^{k}}(t) e^{\left(m^{k}, \tau\right)}+Q(t, \tau),
\end{gathered}
$$

Here in the embedding we need only the terms

$$
\begin{gathered}
M(t, \tau) \equiv \sum_{i=3}^{4} \frac{g(t)}{2} B(t) z_{i}(t)\left(e^{\tau_{i}+\tau_{3}} \sigma_{1}+e^{\tau_{i}+\tau_{4}} \sigma_{2}\right) \\
+\sum_{k=1}^{2} \frac{g(t)}{2} B(t) z_{k}(t)\left(e^{\tau_{k}+\tau_{3}} \sigma_{1}+e^{\tau_{k}+\tau_{4}} \sigma_{2}\right), \\
S(t, \tau) \equiv \sum_{2 \leq|m| \leq N_{w}}^{*} w^{m}(t) e^{(m, \tau)}+\sum_{k=1}^{2} \sum_{1 \leq|m| \leq N_{w}}^{*} w^{e_{k}+m}(t) e^{\left(e_{k}+m, \tau\right)}
\end{gathered}
$$




$$
\begin{gathered}
+\sum_{2 \leq|m| \leq N_{w}: m \in \Gamma_{0}} w^{m}(t) e^{(m, \tau)}+\sum_{k=1}^{2} \sum_{2 \leq|m| \leq N_{w}: m \in \Gamma_{k}} w^{m}(t) e^{(m, \tau)} \\
+\sum_{k=1}^{2} \sum_{j=1}^{2} \sum_{1 \leq\left|m^{k}\right| \leq N_{w}: m \in \Gamma_{k, j}} w^{m^{k}}(t) e^{\left(m^{k}, \tau\right)} .
\end{gathered}
$$

We describe this embedding in details:

$$
\begin{gathered}
M(t, \tau) \equiv \sum_{k=1}^{2} \frac{g(t)}{2} B(t) z_{k}(t)\left(e^{\tau_{k}+\tau_{3}} \sigma_{1}+e^{\tau_{k}+\tau_{4}} \sigma_{2}\right) \\
+\sum_{i=3}^{4} \frac{g(t)}{2} B(t) z_{i}(t)\left(e^{\tau_{i}+\tau_{3}} \sigma_{1}+e^{\tau_{i}+\tau_{4}} \sigma_{2}\right) \\
=\frac{g(t)}{2} B(t)\left[z_{1}(t) e^{\tau_{1}+\tau_{3}} \sigma_{1}+z_{1}(t) e^{\tau_{1}+\tau_{4}} \sigma_{2}+z_{2}(t) e^{\tau_{2}+\tau_{3}} \sigma_{1}\right. \\
+z_{2}(t) e^{\tau_{2}+\tau_{4}} \sigma_{2}+z_{3}(t) e^{2 \tau_{3}} \sigma_{1}+z_{3}(t) e^{\tau_{3}+\tau_{4}} \sigma_{2}+z_{4}(t) e^{\tau_{4}+\tau_{3}} \sigma_{1} \\
\left.\quad+z_{4}(t) e^{2 \tau_{4}} \sigma_{2}\right] \Rightarrow \\
\Rightarrow \widehat{M}(t, \tau)=\frac{g(t)}{2} B(t)\left[z_{1}(t) e^{\tau_{1}+\tau_{3}} \sigma_{1}+z_{1}(t) e^{\tau_{1}+\tau_{4}} \sigma_{2}\right. \\
+z_{2}(t) e^{\tau_{2}+\tau_{3}} \sigma_{1}+z_{2}(t) e^{\tau_{2}+\tau_{4}} \sigma_{2}+z_{3}(t) e^{2 \tau_{3}} \sigma_{1}+z_{3}(t) \sigma_{2} \\
+z_{4}(t) \sigma_{1}+z_{4}(t) e^{2 \tau_{4}} \sigma_{2}
\end{gathered}
$$

(we note, that in $\widehat{M}(t, \tau)$ there are not terms, which contain $e^{\tau_{1}}, e^{\tau_{2}}$ of dimension $|m|=1)$

$$
\begin{aligned}
\widehat{S}(t, \tau) & \equiv \sum_{2 \leq|m| \leq N_{w}}^{*} w^{m}(t) e^{(m, \tau)}+\sum_{k=1}^{2} \sum_{1 \leq|m| \leq N_{w}}^{*} w^{e_{k}+m}(t) e^{\left(e_{k}+m, \tau\right)} \\
& +\sum_{2 \leq|m| \leq N_{w}: m \in \Gamma_{0}} w^{m}(t)+\sum_{k=1}^{2} \sum_{2 \leq|m| \leq N_{w}: m \in \Gamma_{k}} w^{m}(t) e^{\tau_{k}} \\
& +\sum_{k=1}^{2} \sum_{j=1}^{2} \sum_{1 \leq\left|m^{k}\right| \leq N_{w}: m \in \Gamma_{k, j}} w^{m^{k}}(t) e^{\tau_{j}} .
\end{aligned}
$$


After embedding, the right-hand side of the system (17) has the form

$$
\begin{aligned}
& \widehat{G}(t, \tau)=-\frac{\partial}{\partial t}\left[z_{0}(t)+\sum_{i=1}^{4} z_{i}(t) e^{\tau_{i}}+\sum_{2 \leq|m| \leq N_{z}}^{*} z^{m}(t) e^{(m, \tau)}\right. \\
& \left.+\sum_{j=1}^{2} \sum_{1 \leq|m| \leq N_{z}}^{*} z^{e_{j}+m}(t) e^{\left(e_{j}+m, \tau\right)}\right]+\widehat{M}(t, \tau)+\widehat{S}(t, \tau)+Q(t, \tau),
\end{aligned}
$$

moreover, in $\widehat{S}(t, \tau)$ coefficients at $e^{\tau_{1}}, e^{\tau_{2}}$ do not depend on $z_{k}(t), k=1,2$. As indicated in [5], the embedding $G(t, \tau) \rightarrow \widehat{G}(t, \tau)$ will not affect to accuracy of construction of asymptotic solutions of the problem (2), since $\left.\widehat{Z}(t, \tau)\right|_{\tau=\psi(t) / \varepsilon} \equiv$ $\left.Z(t, \tau)\right|_{\tau=\psi(t) / \varepsilon}$.

Theorem 2. Let the conditions 1) and 2) hold and the right hand side $H(t, \tau)=H_{0}(t)+\sum_{i=1}^{4} H_{i}(t) e^{\tau_{i}}+\sum_{2 \leq|m| \leq N_{z}}^{*} H^{m}(t) e^{(m, \tau)}$ $+\sum_{j=1}^{2} \sum_{1 \leq|m| \leq N_{H}}^{*} H^{e_{j}+m}(t) e^{\left(e_{j}+m, \tau\right)} \in U$ of the system (11) satisfies the condition (12). Then the problem (11) with the additional conditions

$$
<\widehat{G}(t, \tau), \chi_{k}(t) e^{\tau_{k}}>\equiv 0 \forall t \in\left[t_{0}, T\right], k=1,2,
$$

where $Q(t, \tau)=Q_{0}(t)+\sum_{k=1}^{4} Q_{k}(t) e^{\tau_{k}}+\sum_{2 \leq|m| \leq N_{z}}^{*} Q^{m}(t) e^{(m, \tau)}$ $\sum_{k=1}^{2} \sum_{1 \leq|m| \leq N_{Q}}^{*} Q^{e_{k}+m}(t) e^{\left(e_{k}+m, \tau\right)}$ is known vector function in the space $U$, is uniquely solvable in $U$.

Proof. Since the right hand side of the system (11) satisfies the condition (11), then this system has a solution in the space $U$ as (16), where $\alpha_{k}(t) \in$ $C^{\infty}\left(\left[t_{0}, T\right], C^{1}\right)$ are still arbitrary functions, $k=1,2$. Subordinate (16) to the initial condition $z\left(t_{0}, 0\right)=z^{*}$. We get $\sum_{k=1}^{2} \alpha_{k}\left(t_{0}\right) \varphi_{k}\left(t_{0}\right)=z_{*}$, where

$$
\begin{gathered}
z_{*}=z^{*}+A^{-1}\left(t_{0}\right) H_{0}\left(t_{0}\right)-\sum_{i=3}^{4}\left[\lambda_{i}\left(t_{0}\right) I-A\left(t_{0}\right)\right]^{-1} H_{i}\left(t_{0}\right) \\
-\frac{\left(H^{e_{1}}\left(t_{0}\right), \chi_{2}\left(t_{0}\right)\right)}{\lambda_{1}\left(t_{0}\right)-\lambda_{2}\left(t_{0}\right)} \varphi_{2}\left(t_{0}\right)-\frac{\left(H^{e_{2}}\left(t_{0}\right), \chi_{1}\left(t_{0}\right)\right)}{\lambda_{2}\left(t_{0}\right)-\lambda_{1}\left(t_{0}\right)} \varphi_{1}\left(t_{0}\right)
\end{gathered}
$$




$$
\begin{gathered}
-\sum_{2 \leq|m| \leq N_{z}}^{*} z^{m}\left(t_{0}\right) e^{(m, \tau)}-\sum_{k=1}^{2} \sum_{1 \leq\left|m^{k}\right| \leq N_{H}}^{*}\left[\left(m^{k}, \lambda\left(t_{0}\right)\right) I\right. \\
\left.-A\left(t_{0}\right)\right]^{-1} H^{m^{k}}\left(t_{0}\right) .
\end{gathered}
$$

Multiplying scalar the equality $\sum_{k=1}^{2} \alpha_{k}\left(t_{0}\right) \varphi_{k}\left(t_{0}\right)=z_{*}$ by $\chi_{j}\left(t_{0}\right)$ and taking into account biorthogonality of the systems $\left\{\varphi_{k}(t)\right\}$ and $\chi_{j}\left(t_{0}\right)$ we find the value $\alpha_{k}\left(t_{0}\right)=\frac{1}{2}\left(z_{*}, \chi_{k}\left(t_{0}\right)\right), k=1,2$.

Now we subordinate the solution (16) to the condition of orthogonality (18). Taking into account the fact that under these conditions, scalar multiplication by the vector functions $\chi_{k}(t) e^{\tau_{k}}$, containing only exponents $e^{\tau_{k}}, k=1,2$, with the conditions (18) is made, in the expressions for $\widehat{G}(t, \tau)$ it is necessary to keep only the terms with exponents $e^{\tau_{1}}$ and $e^{\tau_{2}}$. Then the condition (18) takes the form

$$
\begin{gathered}
<-\frac{\partial}{\partial t}\left(\sum_{k=1}^{2} \alpha_{k}(t) \varphi_{k}(t) e^{\tau_{k}}+\frac{\left(H_{1}(t), \chi_{2}(t)\right)}{\lambda_{1}(t)-\lambda_{2}(t)} \varphi_{2}(t) e^{\tau_{1}}\right. \\
\left.+\frac{\left(H_{2}(t), \chi_{1}(t)\right)}{\lambda_{2}(t)-\lambda_{1}(t)} \varphi_{1}(t) e^{\tau_{2}}\right)+\sum_{k=1}^{2} \sum_{2 \leq|m| \leq N_{w}: m \in \Gamma_{k}} w^{m}(t) e^{\tau_{k}} \\
+\sum_{k=1}^{2} \sum_{j=1}^{2} \sum_{1 \leq\left|m^{k}\right| \leq N_{w}: m \in \Gamma_{k, j}} w^{m^{k}}(t) e^{\tau_{j}}+Q_{1}(t) e^{\tau_{1}} \\
+Q_{2}(t) e^{\tau_{2}}, \chi_{k}(t) e^{\tau_{k}}>\equiv 0, \quad \forall t \in\left[t_{0}, T\right], k=1,2 .
\end{gathered}
$$

By performing the scalar multiplication here, we obtain linear ordinary differential equations for the functions $\alpha_{k}(t)$, involved in the solution (16) of the system (11). Attaching to them the initial conditions $\alpha_{k}\left(t_{0}\right)=\frac{1}{2}\left(z_{*}, \chi_{k}\left(t_{0}\right)\right), k=$ 1,2 , calculated earlier, we find uniquely the functions $\alpha_{k}(t) \in C^{\infty}\left(\left[t_{0}, T\right], C^{2}\right), k=$ 1,2 , and, therefore, we construct the solution (16) in the space $U$ in a unique way. Theorem 2 is proved.

Applying Theorems 1 and 2 to the iteration problems $\left(10_{k}\right)$ (we put the right-hand sides $H^{(k)}(t, \tau)$ of these problems into the space $U$ that is, we replace $H^{(k)}(t, \tau)$ with $\left.\hat{H}^{(k)}(t, \tau) \in U\right)$, we find uniquely their solutions in the space $U$ and construct the series (17). Just as in [5], we prove the following statement.

Theorem 3. Suppose that for the systems (2) the conditions 1)-2) hold. Then for $\varepsilon \in\left(0, \varepsilon_{0}\right]\left(\varepsilon_{0}>0\right.$ is small enough) the system (2) has a unique solution 
$z(t, \varepsilon) \in \mathrm{C}^{1}\left([0, T], C^{2}\right) ;$ moreover

$$
\left\|z(t, \varepsilon)-z_{\varepsilon N}(t)\right\|_{C[0, T]} \leq c_{N} \varepsilon^{N+1},
$$

where $z_{\varepsilon N}(t)$ constriction (at $\left.\tau=\frac{\psi(t)}{\varepsilon}\right) N-$ th partial sum of the series (7) (with coefficients $z_{k}(t, \tau) \in U$ satisfying the iteration problems $\left(10_{k}\right)$ ), and the constant $c_{N}>0$ does not depend on $\varepsilon$ for $\varepsilon \in\left(0, \varepsilon_{0}\right]$.

\section{Construction of a solution of the first iteration problem in the space $U$}

We construct the leading term of the asymptotics of the solution to problem (1) in the case $\gamma^{\prime}(t) \equiv \omega(t)$. Using Theorem 1, we try to find a solution of the first iteration problem $\left(10_{0}\right)$. Since the right hand side $h(t)$ of the system $\left(10_{0}\right)$ satisfies the condition (12), then this system has (due to (16)) a solution of the following form in the space $U$ :

$$
z_{0}(t, \tau)=z_{0}^{(0)}(t)+\sum_{k=1}^{2} \alpha_{k}^{(0)}(t) \varphi_{k}(t) e^{\tau_{k}}
$$

where $z_{0}^{(0)}(t)$ is a solution of the integral system

$$
z_{0}^{(0)}(t)=\int_{t_{0}}^{t}\left(-A^{-1}(t) K(t, s)\right) z_{0}^{(0)}(s) d s-A^{-1}(t) h(t)
$$

$\alpha_{k}^{(0)}(t) \in C^{\infty}\left(\left[t_{0}, T\right], C^{1}\right)$ are arbitrary functions, $k=1,2$. Subordinating (19) to the initial condition $z_{0}\left(t_{0}, 0\right)=z^{0}$, we have

$$
\begin{aligned}
& z_{0}^{(0)}\left(t_{0}\right)+\sum_{k=1}^{2} \alpha_{k}^{(0)}\left(t_{0}\right) \varphi_{k}\left(t_{0}\right)=z^{0} \Leftrightarrow \\
& \Leftrightarrow \quad \sum_{k=1}^{2} \alpha_{k}^{(0)}\left(t_{0}\right) \varphi_{k}\left(t_{0}\right)=z^{0}+A^{-1}\left(t_{0}\right) h\left(t_{0}\right) .
\end{aligned}
$$

Scalar multiplying this equality by $\chi_{j}\left(t_{0}\right)$ and taking into account biorthogonality of the systems $\left\{\varphi_{k}(t)\right\}$ and $\left\{\chi_{j}(t)\right\}$, we find the value $\alpha_{k}^{(0)}\left(t_{0}\right)=$ $\frac{1}{2}\left(z^{0}+A^{-1}\left(t_{0}\right) h\left(t_{0}\right), \chi_{k}\left(t_{0}\right)\right), k=1,2$. To fully calculate the functions $\alpha_{k}^{(0)}(t)$ 
we turn to the next iteration problem $\left(10_{1}\right)$. Putting the solution (17) of the system $\left(10_{0}\right)$, in it, we come to the following system:

$$
\begin{gathered}
L z_{1}(t, \tau)=-\frac{d}{d t} z_{0}^{(0)}(t)-\sum_{k=1}^{2} \frac{d}{d t}\left(\alpha_{k}^{(0)}(t) \varphi_{k}(t)\right) e^{\tau_{k}} \\
+\frac{g(t)}{2}\left(e^{\tau_{3}} \sigma_{1}+e^{\tau_{4}} \sigma_{2}\right) B(t)\left(z_{0}^{(0)}(t)+\sum_{k=1}^{2} \alpha_{k}^{(0)}(t) \varphi_{k}(t) e^{\tau_{k}}\right) \\
+\sum_{j=1}^{2}\left[\frac{\left(K(t, t) \alpha_{j}^{(0)}(t) \varphi_{j}(t)\right)}{\lambda_{j}(t)} e^{\tau_{j}}-\frac{\left(K\left(t, t_{0}\right) \alpha_{j}^{(0)}\left(t_{0}\right) \varphi_{j}\left(t_{0}\right)\right)}{\lambda_{j}\left(t_{0}\right)}\right],
\end{gathered}
$$

(here we use the expression $\left(6_{1}\right)$ for $R_{1} z(t, \tau)$ and take into account the fact that when $z(t, \tau)=z_{0}(t, \tau)$ in the sum $\left(6_{1}\right)$ only terms with $e^{\tau_{1}}$ and $e^{\tau_{2}}$ remain). It is easy to see that the right hand side of the system (21) after its embedding in the space $U$ will look like

$$
\begin{gathered}
H(t, \tau)=-\frac{d}{d t} z_{0}^{(0)}(t)-\sum_{k=1}^{2} \frac{d}{d t}\left(\alpha_{k}^{(0)}(t) \varphi_{k}(t)\right) e^{\tau_{k}} \\
+\frac{1}{2} g(t) B(t) e^{\tau_{3}} \sigma_{1} z_{0}(t)+\frac{1}{2} g(t) B(t) e^{\tau_{4}} \sigma_{2} z_{0}(t) \\
+\frac{1}{2} g(t) B(t) \sigma_{1} \alpha_{1}^{(0)}(t) \varphi_{1}(t) e^{\tau_{1}+\tau_{3}}+\frac{1}{2} g(t) B(t) \sigma_{1} \alpha_{2}^{(0)}(t) \varphi_{2}(t) \\
+\frac{1}{2} g(t) B(t) \sigma_{2} \alpha_{1}^{(0)}(t) \varphi_{1}(t)+\frac{1}{2} g(t) B(t) \sigma_{2} \alpha_{2}^{(0)}(t) \varphi_{2}(t) e^{\tau_{2}+\tau_{4}} \\
+\sum_{j=1}^{2}\left[\frac{\left(K(t, t) \alpha_{j}^{(0)}(t) \varphi_{j}(t)\right)}{\lambda_{j}(t)} e^{\tau_{j}}-\frac{\left(K\left(t, t_{0}\right) \alpha_{j}^{(0)}\left(t_{0}\right) \varphi_{j}\left(t_{0}\right)\right)}{\lambda_{j}\left(t_{0}\right)}\right] .
\end{gathered}
$$

It is assumed here that if $\gamma^{\prime}(t) \equiv \omega(t)$, then only a resonance:

$$
1 \cdot(+i \omega)+1 \cdot\left(-i \gamma^{\prime}\right)=0,1 \cdot(-i \omega)+1 \cdot\left(+i \gamma^{\prime}\right)=0
$$

takes place, therefore only exponentials $e^{\tau_{2}+\tau_{3}}, e^{\tau_{1}+\tau_{4}}$ will be resonant and for them the following embedding: $\left(e^{\tau_{2}+\tau_{3}}\right)^{\wedge}=\left(e^{\tau_{1}+\tau_{4}}\right)^{\wedge}==1$ holds true. The system (21) is solvable in this space if and only if the conditions (12) hold, which in our case take the form

$$
\left(-\frac{d}{d t}\left(\alpha_{k}^{(0)}(t) \varphi_{k}(t)\right)+\frac{\left(K(t, t) \alpha_{k}^{(0)}(t) \varphi_{k}(t)\right)}{\lambda_{k}(t)}, \chi_{k}(t)\right)=0 \Leftrightarrow
$$




$$
\Leftrightarrow 2 \frac{d \alpha_{k}^{(0)}(t)}{d t}=\left(\frac{\left(K(t, t) \varphi_{k}(t)\right)}{\lambda_{k}(t)}-\dot{\varphi}_{k}(t), \chi_{k}(t)\right) \alpha_{k}^{(0)}(t), k=1,2 .
$$

Attaching initial conditions $\alpha_{k}^{(0)}\left(t_{0}\right)=\frac{1}{2}\left(z^{0}+A^{-1}\left(t_{0}\right) h\left(t_{0}\right), \quad, \chi_{k}\left(t_{0}\right)\right), k=$ 1,2 , to this system, we uniquely find the functions

$$
\alpha_{k}^{(0)}(t)=\alpha_{k}^{(0)}\left(t_{0}\right) \mathrm{e}^{\frac{1}{2} \int_{t_{0}}^{t}\left(\frac{\left(K(s, s) \varphi_{k}(t)\right)}{\lambda_{k}(s)}-\dot{\varphi}_{k}(s), \chi_{k}(s)\right) d s}, k=1,2,
$$

therefore, we uniquely calculate the solution (19) of the problem $\left(10_{0}\right)$ in the space $U$. Moreover, the main term of the asymptotics of the solution of the problem (2) is

$$
\begin{gathered}
z_{\varepsilon 0}(t)=z_{0}^{(0)}(t) \\
+\sum_{k=1}^{2} \alpha_{k}^{(0)}\left(t_{0}\right) \mathrm{e}^{\frac{1}{2} \int_{0}^{t}\left(\frac{\left(K(s, s) \varphi_{k}(s)\right)}{\lambda_{k}(s)}-\dot{\varphi}_{k}(s), \chi_{k}(s)\right) d s} \varphi_{k}(t) e^{\frac{1}{\varepsilon} \int_{t_{0}}^{t} \lambda_{k}(\theta) d \theta},
\end{gathered}
$$

where $\alpha_{k}^{(0)}\left(t_{0}\right)=\frac{1}{2}\left(z^{0}+A^{-1}\left(t_{0}\right) h\left(t_{0}\right), \chi_{k}\left(t_{0}\right)\right), k=1,2, z_{0}^{(0)}(t)$ is a solution of the integral system $z_{0}^{(0)}(t)=\int_{t_{0}}^{t}\left(-A^{-1}(t) K(t, s)\right) \times \times z_{0}^{(0)}(s) d s-$ $A^{-1}(t) h(t)$.

\section{Conclusion}

Analysis of the function $z_{\varepsilon 0}(t)$ shows that when we remove from a differential system of the type $(1)(K(t, s) \equiv 0)$ to an integro-differential system $(K(t, s) \neq 0)$, only the diagonal kernel $K(t, t)$. has an effect on the main term of the asymptotics.

If $K(t, t) \equiv 0$, then the main terms of asymptotics of the differential and integro-differential problems coincide. We also note that in the case $\gamma^{\prime}(t) \equiv$ $\omega(t)$ the resonant exponents are not involved in construction of the main term of the asymptotics $z_{\varepsilon 0}(t)$. In other cases of resonance (for example, in the case $\gamma^{\prime}=2 \omega$ ) the leading term of the asymptotics will already be affected by the rapidly oscillating term in (1).

\section{Acknowledgements}

This work is supported by the grant AP05133858 "Contrast structures in singularly perturbed equations and their applications in the theory of phase transitions" by Ministry of Education and Science of the Republic of Kazakhstan. 


\section{References}

[1] Yu.L. Daletskiy and M.G. Krein, Stability of Solutions of Differential Equations in Banach Space, Moscow, Nauka (1970).

[2] Yu.L. Daletskiy, Asymptotic method for some differential equations with oscillating coefficients, DAN SSSR, 143, No 5 (1962), 1026-1029.

[3] S.F. Feschenko, N.U. Shkill and L.D. Nikolenko, Asymptotic Methods in the Theory of Linear Differential Equations, Kiev, Naukova Dumka (1966).

[4] B.T. Kalimbetov and V.F. Safonov, Integro-differentiated singularly perturbed equations with fast oscillating coefficients, Bulletin of KarSU, Ser. Mathematics, 94, No 2 (2019), 33-47.

[5] S.A. Lomov, Introduction to General Theory of Singular Perturbations, Moscow, Nauka (1981).

[6] S.A. Lomov and I.S. Lomov, Foundations of Mathemathical Theory of Boundary Layer, Publishing House of Moscow State University (2011).

[7] A.D. Ryzhih, Asymptotic solution of linear differential equation with rapidly oscillating coefficient, Trudy MPEI, 357 (1978), 92-94.

[8] A.D. Ryzhih, Application of regularization method for an equation with rapidly oscillating coefficients, In: Mater. All-Union. Conf. by Asympt. Methods, Science, Alma-Ata (1979), 64-66.

[9] V.F. Safonov and A.A. Bobodzhanov, Course of Higher Mathematics. Singularly Perturbed Problems and Regularization Method: Textbook, Moscow, Publishing House of MPEI (2012). 
